\title{
Low-cost glass ionomer cement as ART sealant in permanent molars: a randomized clinical trial
}

\section{Daniela HESSE(a) \\ Clarissa Calil BONIFÁCIO(b) \\ Camila de Almeida Brandão GUGLIELMI(a) \\ Carolina da FRANCA(c) \\ Fausto Medeiros MENDES(a) \\ Daniela Prócida RAGGIO(a)}

(a) Universidade de São Paulo - USP, Dental School, Orthodontics and Pediatric Dentistry Department, São Paulo, SP, Brazil.

(b)Academic Centre for Dentistry Amsterdam - ACTA, Department of Conservative Dentistry, Amsterdam, The Netherlands.

(c) Universidade de Pernambuco - UPE, Dental School, Department of Social Dentistry, Recife, PE, Brazil.

Declaration of Interests: The authors certify that they have no commercial or associative interest that represents a conflict of interest in connection with the manuscript.

Corresponding Author:

Daniela Hesse

E-mail: dhesse@usp.br

DOI: 10.1590/1807-3107BOR-2015.vol29.0063

Submitted: Out 15, 2014

Accepted for publication: Jan 02, 2015

Last revision: Apr 23, 2015

\begin{abstract}
Clinical trials are normally performed with well-known brands of glass ionomer cement (GIC), but the cost of these materials is high for public healthcare in less-affluent communities. Given the need to research cheaper materials, it seems pertinent to investigate the retention rate of a low-cost GIC applied as atraumatic restorative treatment (ART) sealants in two centers in Brazil. Four hundred and thirty-seven 6-to-8-year-old schoolchildren were selected in two cities in Brazil. The children were randomly divided into two groups, according to the tested GIC applied in the first permanent molars. The retention rate was evaluated after 3, 6 and 12 months. Kaplan-Meier survival analysis and the log-rank test were performed. The variables were tested for association with sealant longevity, using logistic regression analyses $(\alpha=5 \%$ ). The retention rate of sealants after 12 months was $19.1 \%$. The high-cost GIC brand presented a 2-fold-more-likely-to-survive rate than the low-cost brand ( $p<0.001)$. Significant difference was also found between the cities where the treatments were performed, in that Barueri presented a higher sealant survival rate than Recife $(\mathrm{p}<0.001)$. The retention rate of a low-cost GIC sealant brand was markedly lower than that of a well-known GIC sealant brand.
\end{abstract}

Keywords: Dental Atraumatic Restorative Treatment; Glass Ionomer Cements; Pit and Fissure Sealants.

\section{Introduction}

Atraumatic Restorative Treatment (ART) is a preventive and restorative approach to managing dental caries. It includes sealing caries-prone pits and fissures, ${ }^{1}$ and excavating cavitated dentine caries with hand instruments, and then restoring the cavity., ${ }^{2,3}$ This approach was introduced to provide dental care to less-affluent populations, making caries management possible not only in dental offices, but also in outreach situations. ${ }^{4}$ In addition to using ART in cavitated lesion management, the sealing of incipient enamel lesions seems beneficial, since occlusal surfaces with unsealed enamel-restricted lesions are four times more likely to develop into a dentin lesion than those sealed after 3 years of assessment. ${ }^{2}$

Glass ionomer cement (GIC) exhibits good properties for restorative and preventive dentistry, ${ }^{5}$ making this material the current choice for ART. ${ }^{6}$ High-viscous GICs were specially developed for ART and have the main advantage of improved mechanical properties, resulting in 
long-lasting sealants and restorations. ${ }^{7}$ Bonifácio $e t$ al. ${ }^{8}$ evaluated the physical-mechanical properties of six high-viscous GIC brands, and concluded that Maxxion R and Hi-Dense presented satisfactory performance in the majority of tests, compared with the two top GIC brands for ART (Fuji IX and Ketac Molar Easymix). In a clinical trial, Bonifácio et al. ${ }^{9}$ investigated the survival rate of occlusoproximal restorations made with three different GICs (Fuji IX, Maxxion R and Hi-Dense), and found no differences in the survival rates of the three materials. In addition to showing good performance in these earlier studies, Maxxion R has the benefit of costing less than $25 \%$ of the price of Fuji IX or Ketac Molar Easymix, thus enabling the use of high-viscous GIC for public healthcare in developing countries.

To the best of our knowledge, there are no studies evaluating the performance of Maxxion $\mathrm{R}$ as a pit and fissure sealant. Given the need to research cheaper materials, it seems pertinent to investigate the retention rate of a low-cost GIC applied as an ART sealant in two centers in Brazil.

\section{Methodology Study design}

This was a multicenter study carried out in two cities of two different states in Brazil: Barueri (state of São Paulo - southeast region) and Recife (state of Pernambuco - northeast region). The study design was a split-mouth randomized blind trial with a negative control, reported following the checklist of the CONSORT guidelines (i.e., Consolidated Standards of Reporting Trials).

\section{Setting and study participants}

Barueri is a city located in the southeast region of Brazil, with a population of 240,656 inhabitants. The human development index (HDI) is 0.80 and the child mortality rate is 8.5 per thousand births. ${ }^{10}$ Recife is a city located in the northeast region of Brazil, with a population of 1,537,704 habitants. The HDI is 0.77 and the child mortality rate is 13.90 per thousand births. ${ }^{10}$

The study was approved by the Local Research Ethics Committee (protocol 190/08 - School of
Dentistry, Universidade de São Paulo - USP) prior to its commencement.

The sample size was calculated based on a minimally important difference of $20 \%$ in the success rates between treatment and control groups, over a period of two years, a of $5 \%$ and a power of $80 \%$. A minimum sample of 162 children in each city was required. A total of 2,000 public schoolchildren aged 6-8 years old were examined in each city. We selected 187 children in Barueri and 250 in Recife ( $N=437$ ), who fulfilled the inclusion criteria and had a written consent from parents or legal guardians. The inclusion criteria were: presence of four first permanent molars without gingival tissue covering the occlusal surface, with no clinically detectable carious lesions in the dentine, evaluated using ICDAS criteria (scores 0, 1 and 2), ${ }_{1}^{11}$ cooperative behavior, and in good physical and medical health.

The operators were four final-year Dutch dental students previously trained and calibrated in ICDAS criteria ${ }^{11}$ for the assessment of caries, and in Nyvad criteria $^{12}$ for the assessment of lesion activity. The examiners underwent a training process comprised of four hours of specific training, and calibration sessions with a "benchmark examiner," using clinical slides. Afterwards, hands-on training was also given, which included the evaluation of 30 molars not included in our reserach sample.

The operators were also trained to apply ART sealants, including the mixing of GICs, according to the manufacturer's protocol. A tryout week was included to allow them to familiarize themselves with local conditions, ICDAS assessment and sealant techniques, before initiating the treatments. During this week, 30 patients not included in the sample were treated. The operators were split into two pairs, and were assisted by each other. All treatments were performed on the schools premises, without using a dental chair or other facilities from a dental office. The diagnosis and treatment procedures were performed with the aid of a headlight, and a manual syringe was used to dry the teeth for caries diagnosis and evaluation.

In the actual study, the patients' mouth was split vertically into left and right sides. Simple randomization was used and the side of the mouth 
to be treated was assigned to either the left or the right, according to a random list generated by computer software. The four first permanent molars were studied; two were sealed with either one brand (Fuji XI, GC Europe, Leuven, Belgium) or another brand (Maxxion R, FGM, Joinville, Brazil) of GIC (test molars), and the other two remaining molars, with no sealant (control molars), controlled only by tooth brushing. Another random list was used to assign the schoolchildren to one of the four operators, as well as to the GIC brands used in this study (Fuji IX, or Maxxion R). The patients and caregivers were blinded to the GIC brand used, and four independent dentists, who were not involved in the research, and who had no prior contact with the operators, were invited to carry out the randomization step. The data regarding caries assessment of all first permanent molars were not used in this assessment (of "retention" results), but will be used in a future evaluation of "caries lesions" results.

\section{Intervention}

Both GICs used in this trial were handmix versions, and were mixed according to the manufacturer's instructions (powder/liquid ratio 1:1); surface protection was performed by applying a thin layer of petroleum jelly. The clinical procedures for sealant application followed the steps proposed by Frencken et al.:13 a) remove biofilm and food debris with a toothbrush; $b$ ) isolate the field with cotton wool rolls; c) clean pits and fissures with cotton pellets and water; d) condition with GIC liquid for 20 seconds; e) rinse the occlusal surface with three wet cotton wool pellets; f) dry the occlusal surface with three dry cotton wool pellets; g) place GIC on the occlusal surface; h) press GIC firmly using a gloved index finger with petroleum jelly (press-finger technique); i) remove excess GIC and wait for GIC to set (approximately three minutes); j) check the occlusion and adjust when necessary; k) apply petroleum jelly; l) instruct the patient to avoid eating for at least one hour.

\section{Evaluation}

The follow-up involved a post-treatment evaluation of patients after 3, 6 and 12 months, performed by one independent trained and calibrated examiner at each center. The examiners were trained for sealant retention criteria, ${ }^{14}$ ICDAS $^{11}$ and Nyvad criteria. ${ }^{12}$ They underwent four hours of specific training and calibration sessions with a "benchmark examiner," using clinical slides, followed by hands-on training that included the evaluation of 20 children with sealants.

The examiners were blinded to the material used. The sealants were evaluated on their retention rate, according to the following criteria: 0 - fully present sealant; 1- partially present sealant and 2- absent sealant. ${ }^{14}$ Score 0 was considered as a "success," whereas scores 1 and 2 were considered as a "failure."

\section{Statistical analysis}

Kappa intra- and interexaminer values were calculated for caries and sealant assessment. The intraobserver reliability was performed on 65 patients (15\% of the sample), within a 1-week interval. Kaplan-Meier survival analysis was performed using STATA 11.2 software (StataCorp, College Station, USA) on the censored data for sealant survival. The difference between survival curves was determined by the log-rank test.

The effects of the GIC brand, operator, city (Barueri/Recife), mouth side (left/right), jaw (upper/lower), gender (male/female) and age (years) of the patients were evaluated after the 12-month assessment period, using logistic regression analysis, with MLwiN 2.10 software (Centre for Multilevel Modeling, University of Bristol, Bristol, United Kingdom). All significant differences were detected at a $95 \%$ confidence level.

Trial Registration: Registro Brasileiro de Ensaios Clínicos (REBEC): RBR-2nwk89

\section{Results}

The intraexaminer agreement values for ICDAS and Nyvad criteria ranged from 0.85 to 0.94 , whereas the interexaminer values ranged from 0.70 to 0.90 . The intraexaminer agreement values for sealant retention evaluation were 0.90 in Recife and 0.98 in Barueri, whereas the interexaminer value was 0.97 .

Of the 437 selected children (mean age $7 \pm 0.8$ years old) who participated in the study, 229 (52.4\%) were male. The children were randomly allocated; 201 (46\%) 
had molars sealed with Maxxion R, and 236 (54\%), with Fuji IX; and 216 (49.4\%) were on the right side, and $221(50.6 \%)$, on the left side.

The lost-to-follow-up percentage after 12 months was $10.7 \%$, and was equally distributed between the two groups of GIC brands. A CONSORT flow diagram of the participants' progress through the trial phases is described in Figure 1.

The cumulative survival of sealants was $19.1 \%$ after 12 months, with the highest rate of failure in the 3-month evaluation (Table 1). The survival curves, with censored data, are presented in Figures 2-3. The log-rank test indicated a statistically significant difference between the GIC brands ( $p<0.001)$, in that Fuji IX presented a higher survival rate than Maxxion R (Figure 2). A significant difference was also found between the cities where the treatments were performed, in that Barueri presented a higher sealant survival rate than Recife ( $\mathrm{p}<0.001$ - Figure 3$)$.

The results of the univariate analysis (Table 2 ) showed that the GIC brand, the jaw, the city and the operator had an impact on the survival rate of the sealants. The final adjusted model of the relationship between associated factors revealed that the sealants made with GIC Fuji IX were twice as likely to survive than those made with Maxxion R. Sealants applied on the upper jaw showed better performance $(p=0.018)$, and the operators had an effect, in that the sealants applied by one of the operators presented a significantly lower retention rate than the sealants applied by the other three $(p=0.02)$. Additionally, the sealants applied in Barueri had a $75 \%$ greater chance of survival than those applied in Recife (Table 2).

\section{Discussion}

The present study evaluated the retention rate of sealants applied using the ART approach. It differs from other studies because it investigated the survival rate of Maxxion R, a low-cost high-viscous GIC, compared with Fuji IX, a well-known GIC, used traditionally in clinical trials. ${ }^{15,16}$ Moreover, it was carried out in two distinct cities, with distinctive socioeconomic and demographic characteristics and climate factors.

The design selected for this study was split-mouth with a negative control. This design was selected because of, not only the survival rate, but also the preventive effect of the sealants (data under investigation) could be assessed, an outcome that requires a longer period of evaluation.

Clinical trials are commonly conducted using the most trustworthy and expensive GIC. ${ }^{15,16}$ The high cost of GIC is a major disadvantage that makes treatment in the public health units of developing countries unfeasible. The Brazilian brand used in this study (Maxxion R) has the advantage of costing less than $25 \%$ of the price of the GIC used in most of the ART studies (Fuji IX and Ketac Molar), without compromising the mechanical and physical properties of GICs, ${ }^{7}$ or the longevity of restorative treatments. ${ }^{9}$ In the present study, the GIC brands showed a significant difference in survival rate, in that Maxxion $\mathrm{R}$ was twice as likely to fail than Fuji IX. The explanation for the better performance of Fuji IX could be attributed to its increased powder-liquid ratio,factor which resulted in better physical-mechanical properties of this material after in vitro tests. ${ }^{8,17}$

Interestingly, our results differ from those of Bonifácio et al., ${ }^{9}$ who reported similar survival rates between Maxxion R and the tested "gold standard" GIC (Fuji IX). However, they investigated the survival rate of occlusoproximal restorations, and the main reason that these types of fillings fail is restoration fracture or loss. This is because they are placed in areas submitted to intense stress, ${ }^{18}$ which may impair GIC performance, even the most reputable ones. ${ }^{19}$ This factor may also explain the lack of difference observed among the GICs used for occlusoproximal restorations. Nevertheless, since Maxxion R costs substantially less than other GICs, and presents more failures, further studies should be performed to ascertain if Maxxion $\mathrm{R}$ is worthwhile using, insofar as its replacement plus retreatment may not make it cost-effective.

One interesting result of our study was the difference between the cities where the sealants were applied, in that the sealants used in Barueri were 75\% longer lasting than the same sealants used in Recife. We hypothesize that the difference in the sealant retention rates between the cities can be attributed to the weather conditions, considering that the treatments were performed in autumn 2010, when the average temperature in Recife was about $30^{\circ} \mathrm{C}$, and the air humidity, $80 \%$. In Barueri 


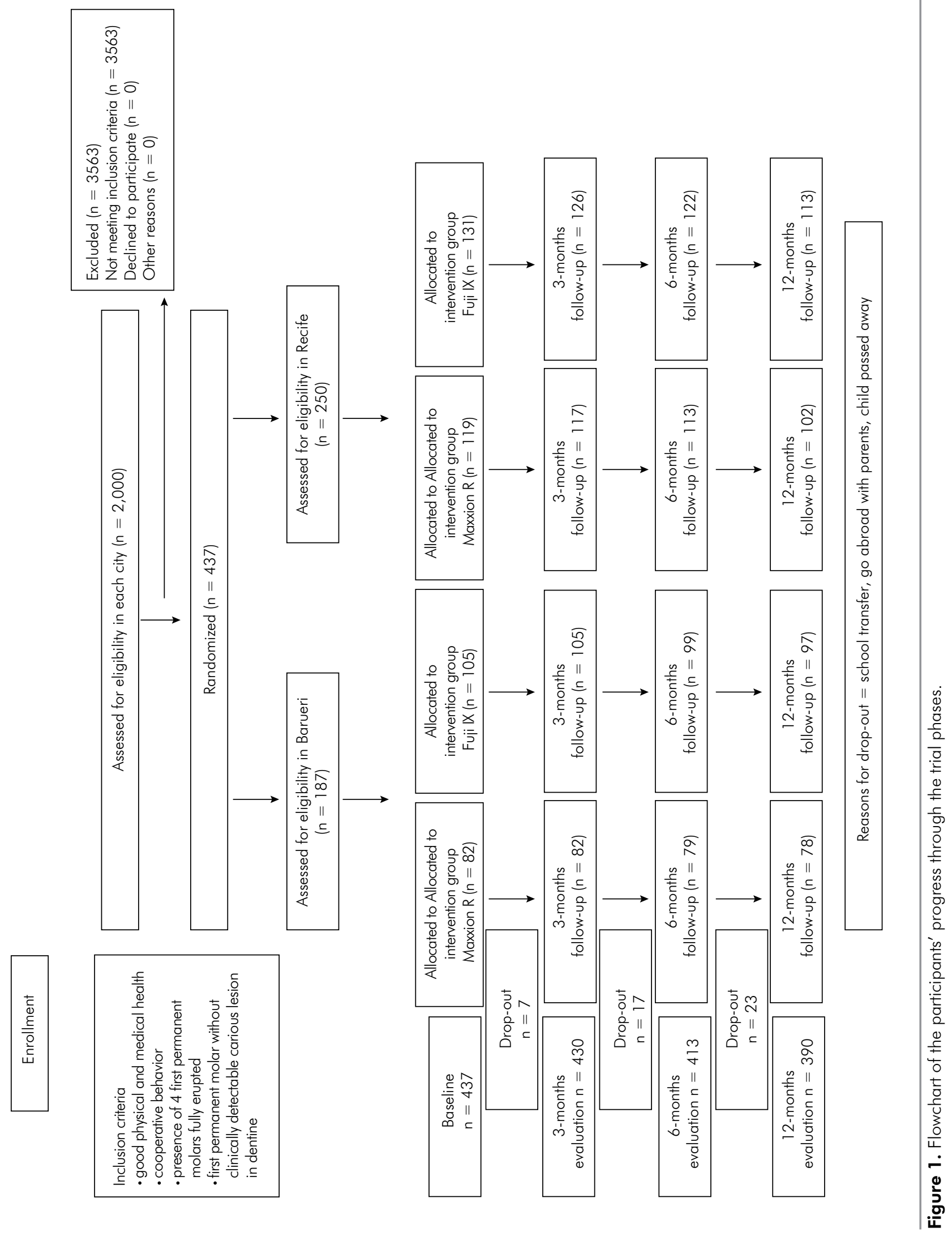


Low-cost glass ionomer cement as ART sealant in permanent molars: a randomized clinical trial

Table 1. Cumulative survival rates (\%) of ART sealants placed in permanent molars, by GICs tested over a 12-month period.

\begin{tabular}{lcccccccc}
\hline & \multicolumn{4}{c}{ Fuji IX } \\
\cline { 2 - 9 } Interval months & N sealants & N censored & N failed & \% failed & N sealants & N censored & N failed & \% failed \\
\hline 3 months & 460 & 12 & 188 & 40.8 & 400 & 2 & 323 & 80.7 \\
6 months & 270 & 2 & 60 & 22.2 & 74 & 3 & 43 & 58.1 \\
12 months & 202 & 8 & 68 & 33.6 & 31 & 0 & 13 & 41.9 \\
\hline
\end{tabular}

$\mathrm{N}$ sealants: number of sealants at start of interval, $\mathrm{N}$ censored: number of sealants censored during interval, $\mathrm{N}$ failed: number of sealants failed at end of interval.

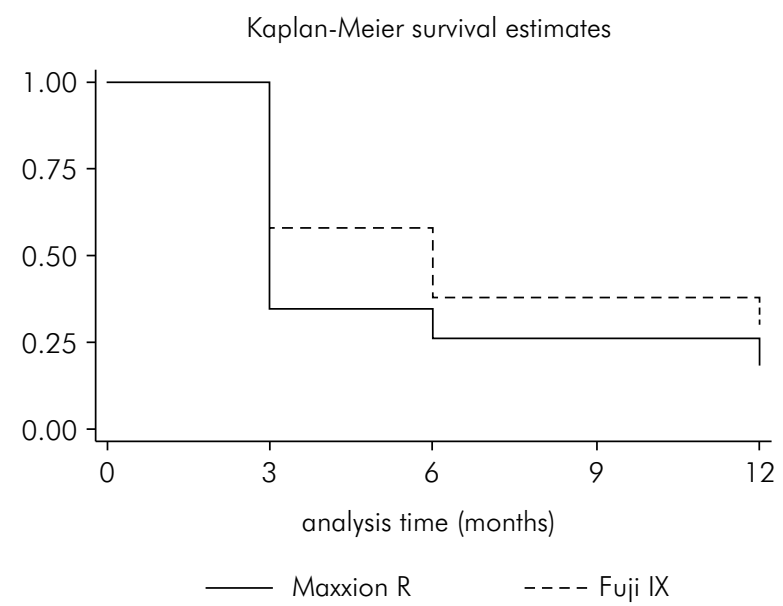

Figure 2. Survival curves of the sealants in regard to the materials used. Log-rank, p-value $<0.001$.

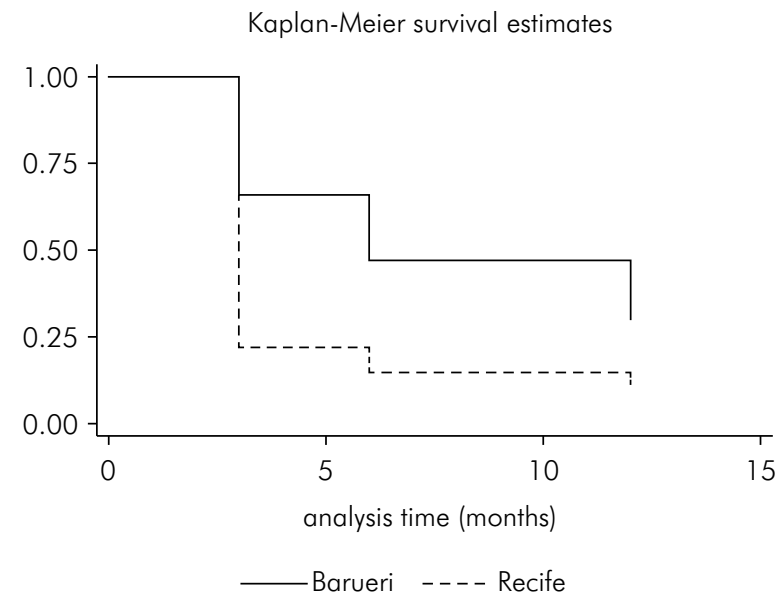

Figure 3. Survival curves of the sealants in regard to the city where the treatments were performed. Log-rank, $p$-value $<0.001$
Table 2. Univariate and multiple logistic regression analysis of sealant retention and associated factors after 12-month assessment.

\begin{tabular}{|c|c|c|c|c|}
\hline & $\begin{array}{c}\text { Crude OR } † \\
95 \% \mathrm{Cl} \neq\end{array}$ & p-value & $\begin{array}{c}\text { Adjusted OR } \dagger \\
95 \% \mathrm{Cl} \ddagger\end{array}$ & $p$-value \\
\hline \multicolumn{5}{|l|}{ GIC brand } \\
\hline Fuji IX & 1.95 & $<0.001$ & 2.05 & $<0.001^{*}$ \\
\hline Maxxion R & $1.63-2.33$ & & $1.67-2.52$ & \\
\hline \multicolumn{5}{|l|}{ Gender } \\
\hline male & 0.91 & 0.27 & - & - \\
\hline female & $0.77-1.08$ & & & \\
\hline \multicolumn{5}{|l|}{ Mouth side } \\
\hline right & 1.07 & 0.44 & - & - \\
\hline left & $0.90-1.26$ & & & \\
\hline \multicolumn{5}{|l|}{ Jaw } \\
\hline upper & 1.11 & 0.03 & 1.15 & $0.01^{*}$ \\
\hline lower & $1.01-1.23$ & & $1.02-1.29$ & \\
\hline \multicolumn{5}{|l|}{ City } \\
\hline Recife & 0.23 & $<0.001$ & 0.25 & $0.003^{*}$ \\
\hline Barveri & $0.10-0.54$ & & $0.10-0.62$ & \\
\hline \multicolumn{5}{|l|}{ Operator } \\
\hline operator 1 & & 0.004 & 0.93 & $0.02^{*}$ \\
\hline \multirow[t]{2}{*}{ operator 2} & 0.77 & & $0.57-1.53$ & \\
\hline & $0.49-1.22$ & & & \\
\hline \multirow[t]{2}{*}{ operator 3} & 0.67 & & 0.66 & \\
\hline & $0.42-1.07$ & & $0.38-1.13$ & \\
\hline \multirow[t]{2}{*}{ operator 4} & 0.73 & & 0.75 & \\
\hline & $0.61-0.87$ & & $0.61-0.92$ & \\
\hline \multicolumn{5}{|l|}{ Age } \\
\hline 6 years old & 0.89 & 0.38 & - & - \\
\hline 7 years old & $0.75-1.06$ & & & \\
\hline \multirow[t]{2}{*}{8 years old } & 0.91 & & - & - \\
\hline & $0.77-1.09$ & & & \\
\hline
\end{tabular}

$+\mathrm{OR}=$ Odds ratio

$\ddagger \mathrm{Cl}=$ Confidence interval.

* Denotes statistical significance $(p<0.05)$. 
the climatic conditions were an average temperature of $20^{\circ} \mathrm{C}$, and air humidity of $60 \%$, in the period in which the sealants were applied. ${ }^{20}$ Although the GIC bonding mechanism to the tooth structure is not completely clear, it is known that chemical adhesion is achieved by an interaction between the carboxyl groups of the polyacids and the hydroxyapatite. ${ }^{21}$ If the setting reaction of the material is shortened by the warm weather, less polyacrylic acid is available to interact with tooth structures, leading to a lower number of cross-links and lower wettability of the material. Furthermore, the high temperature experienced in Recife may have interfered in the GIC setting; this could explain the worse retention of sealants in this city.

The authors of the present study also hypothesize that this difference in the sealant survival rate between the cities may be related to differences in socioeconomic indexes and caries prevalence of the population of both cities. ${ }^{22}$ Investigations report that oral health is associated with social and economic indicators, insofar as a lower socioeconomic population has higher levels of the disease, and, therefore, greater treatment needs. ${ }^{23}$ Moreover, it is known that patients with higher caries risk present decreased restoration longevity. ${ }^{24}$ Recife has higher caries prevalence: $47.3 \%$ of the 5-year-old children are caries-free, whereas the percentage in Barueri is $68.3 \% .^{22}$ The socioeconomic indexes are also distinct in the two cities: Barueri has a poverty incidence of $45.46 \%$, whereas the percentage in Recife is $52.50 \%{ }^{25}$ Furthermore, per capita income in Barueri is twice as high as in Recife. ${ }^{11}$ All these diverse features may explain the difference in the survival rate of sealants, according to the city where the treatments were performed.

Individual differences may also occur between different operators. Méjàre and $\mathrm{Mjör}^{26}$ reported an "operator effect" after a one-year assessment; in this case, one operator performed significantly worse than the other two operators. The present study also indicated an operator effect, insofar as the sealants applied by one operator presented a significantly lower retention rate than the sealants applied by the other three operators.

The ART technique involves a number of clinical steps to be followed rigorously in order to assure long-lasting treatments. Unfavorable operator/assistant-dependent factors, such as poor surface conditioning, inappropriate GIC manipulation, unsatisfactory insertion of the material in the cavity or tooth surface, and lack of surface protection, may result in the poor survival results of the ART treatments. ${ }^{27}$ In the present study, all the operators had the same experience in treating patients (all operators were final-year students) and had conducted comprehensive training, not only in applying ART sealants, but also in handling GICs; therefore, the authors presuppose that the difference related to a specific operator is probably related to his/her individual skills.

Our results showed a cumulative sealant retention rate of $19.1 \%$ after the 12 -month assessment. This finding is in disagreement with others in which success rates of $90 \%,{ }^{3} 72 \%,{ }^{2}$ and $42 \%{ }^{28}$ have been reported after one year. The discrepancy between our findings and those previously reported could be a question of defining "success." To the authors of this study, only those sealants that exhibited complete retention of GIC on the occlusal surface of the evaluated molars can be termed as successful. In contrast, in the other studies mentioned, both partially and fully retained sealants were considered as successful, a categorization that increases the success rate of the sealant. If we had considered both categories of complete and partial retention as successful in our study, the success rate of the sealants would have risen to $48.6 \%$. Nonetheless, the choice of considering only fully retained sealants as successful is supported by the current theory that the complete clinical retention of sealant materials should be contemplated as the cutoff for clinical success. ${ }^{29}$

Some limitations are ascribed to our study. The operators were not blinded to the GIC used; however, in an attempt to offset this drawback, they received the information about what material that they would be using, only after the patient was prepared to receive treatment. Nonetheless, although the performance bias was controlled, concealment of the allocation could not be totally assured, since the dentist responsible for allocating the children in each treatment group used a numerical list of randomization, and, therefore, had information about the material to be used. For this reason, we invited four independent dentists not involved in 
the research and having no prior contact with the operators to perform this step. This way, no operator would be benefited, and the deficiency in concealing the allocation would be minimized.

Another limitation that should be pointed out is a patient-related factor that may influence the survival of ART treatments. It is known that post-restoration consumption of "hard consistency" food can negatively influence the longevity of ART treatments. ${ }^{30}$ The patients in our study were instructed not to eat for one hour after the sealant was placed, but there was no way that we could supervise them, and given the young age of the patients enrolled in our investigation, we cannot guarantee that this request was followed rigorously.

The most import characteristic of GIC is considered to be the therapeutic and cariespreventive effect of this material. ${ }^{15}$ It is known that the sealing of pits and fissures is an effective cariespreventive approach. In addition, the benefits of keeping caries lesions from developing on occlusal surfaces, particularly in children, have already been demonstrated by previous investigations. ${ }^{1,5,6}$ However, knowing the retentive rate of GIC sealants is crucial to understanding the possible association that can interfere with the outcome of "caries lesion presence or progression," and to guiding clinicians in choosing their materials on a daily basis.

\section{References}

1. Frencken JE, Makoni F, Sithole WD. Atraumatic restorative treatment and glass-ionomer sealants in a school oral health programme in Zimbabwe: evaluation after 1 year. Caries Res. 1996;30(6):428-33.

2. Frencken JE, Makoni F, Sithole WD, Hackenitz E. Three-year survival of one-surface ART restorations and glass-ionomer sealants in a school oral health programme in Zimbabwe. Caries Res. 1998;32(2):119-26.

3. Frencken JE, Makoni F, Sithole WD. ART restorations and glass ionomer sealants in Zimbabwe: survival after 3 years. Community Dent Oral Epidemiol. 1998 Dec;26(6):372-81.

4. Frencken JE, Holmgren CJ. Caries management through the Atraumatic Restorative Treatment (ART) approach

\section{Conclusion}

The results of this study indicate that the retention rate of a low-cost GIC sealant brand is markedly lower than the retention of a well-known GIC brand sealant. Moreover, factors such as the city where the sealant was applied and an operator effect can be associated with sealant failures.

\section{Acknowledgements}

We would like to thank the participants of the Pediatric Dentistry Post-Graduation Seminar from Faculdade de Odontologia da Universidade de São Paulo - FOUSP for their critical comments. We would like to thank the schoolchildren, their parents and the staff of the schools and the health service of Barueri, especially Alberto Luiz Ferreira Kesselring, Andrei Alvaro Barrichello Chaves and Fernanda Michelotti Cajado, for their kind assistance in conducting this study. We are very grateful to Malou Schoenmakers, Nicole Sluitman, Kristel Filius and Marieke Herman, for their dedication during the operative phase. We would like to express our gratitude to GC Europe for kindly donating the materials used in this study. We would like to thank the Coordenação de Aperfeiçoamento de Pessoal de Nivel Superior (CAPES) and Fundação de Amparo à Pesquisa do Estado de São Paulo (FAPESP). The study was partially supported by the Conselho Nacional de Desenvolvimento Científico e Tecnológico (CNPq) no. 472882/2010-4. Dr. Daniela Raggio receives CNPq Research Productivity Schoolarship."

and glass-ionomers: update 2013. Braz Oral Res. 2014 Jan-Feb;28(1):5-8.

5. Mickenautsch S, Yengopal V, Banerjee A. Atraumatic restorative treatment versus amalgam restoration longevity: a systematic review. Clin Oral Investig. 2010 Jun;14(3):233-40.

6. van 't Hof MA, Frencken JE, van Palenstein Helderman WH, Holmgren CJ. The atraumatic restorative treatment (ART) approach for managing dental caries: a meta-analysis. Int Dent J. 2006 Dec;56(6):345-51.

7. de Amorim RG, Leal SC, Frencken JE. Survival of atraumatic restorative treatment (ART) sealants and restorations: a metaanalysis. Clin Oral Investig. 2012 Apr;16(2):429-41. 
8. Bonifácio CC, Kleverlaan CJ, Raggio DP, Werner A, de Carvalho RC, van Amerongen WE. Physical-mechanical properties of glass ionomer cements indicated for atraumatic restorative treatment. Aust Dent J. 2009 Sep;54(3):233-7.

9. Bonifácio CC, Hesse D, Raggio DP, Bönecker M, van Loveren $C$, van Amerongen WE. The effect of GIC-brand on the survival rate of proximal-ART restorations. Int J Paediatr Dent. 2013 Jul;23(4):251-8.

10. Instituto Brasileiro de Geografia e Estatística [homepage]. Brasília (DF): Instituto Brasileiro de Geografia e Estatística; 2010 [cited 2014 Jun 24]. Available from: http://www.ibge.gov.br/home/default.php.

11. Ismail AI, Sohn W, Tellez M, Amaya A, Sen A, Hasson H, et al. The International Caries Detection and Assessment System (ICDAS): an integrated system for measuring dental caries. Community Dent Oral Epidemiol. 2007 Jun;35(3):170-8.

12. Nyvad B, Machiulskiene V, Baelum V. Reliability of a new caries diagnostic system differentiating between active and inactive caries lesions. Caries Res. 1999 Jul-Aug;33(4):252-60.

13. Frencken JE, Songpaisan Y, Phantumvanit P, Pilot T. An atraumatic restorative treatment (ART) technique: evaluation after one year. Int Dent J. 1994 Oct;44(5):460-4.

14. Oba AA, Dülgergil T, Sönmez IS, Doğan S. Comparison of caries prevention with glass ionomer and composite resin fissure sealants. J Formos Med Assoc. 2009 Nov;108(11):844-8.

15. Franca C, Colares V, van Amerongen E. Two-year evaluation of the atraumatic restorative treatment approach in primary molars class I and II restorations. Int J Paediatr Dent. 2011 Jul;21(4):249-53.

16. Mickenautsch S. How well are GIC product labels related to current systematic review evidence? Dent Update. 2011 Nov;38(9):634-8, 641-2, 644.

17. Shintome LK, Nagayassu MP, Di Nicoló R, Myaki SI. Microhardness of glass ionomer cements indicated for the ART technique according to surface protection treatment and storage time. Braz Oral Res. 2009 Oct-Dec;2(4):439-45.

18. van Gemert-Schriks MC, van Amerongen WE, ten Cate JM, Aartman IH. Three-year survival of single- and two-surface ART restorations in a high-caries child population. Clin Oral Investig. 2007 Dec;11(4):337-43.

19. Raggio DP, Hesse D, Lenzi TL, Guglielmi CAB, Braga MM. Is Atraumatic restorative treatment an option for restoring occlusoproximal caries lesions in primary teeth?
A systematic review and meta-analysis. Int J Paediatr Dent. 2013 Nov;23(6):435-43.

20. Instituto Nacional de Meteorologia [homepage]. Brasília (DF): Instituto Nacional de Meteorologia; 2010 [cited 2014 Jul 13]. Available from: http://www.inmet.gov.br/sim/ gera_graficos.php

21. Wilson AD, Prosser H, Powis DM. Mechanism of adhesion of polyelectrolyte cements to hydroxyapatite. J Dent Res. 1983 May;62(5):590-2.

22. Brasil. Ministério da Saúde [homepage]. SB Brasil: Pesquisa Nacional de Saúde Bucal 2010 - resultados principais. Ministério da Saúde; 2010 [cited 2014 Dec 5] Available from: http://bvsms.saude.gov.br/bvs/publicacoes/pesquisa_ nacional_saude_bucal.pdf

23. van de Sande FH, Opdam NJ, Rodolpho PA, Correa MB, Demarco FF, Cenci MS. Patient risk factors' influence on survival of posterior composites. J Dent Res. 2013 Jul;92(7 Suppl):78S-83S.

24. CorreaMB,PeresMA,PeresKG,HortaBL, Barros AJ,DemarcoFF. Do socioeconomic determinants affect the quality of posterior dental restorations? A multilevel approach. J Dent. 2013 Nov;41(11):960-7.

25. Instituto Brasileiro de Geografia e Estatística [homepage]. Censo Demográfico 2000 e Pesquisa de Orçamentos Familiares - POF 2008/2009. [cited 2014 Dec 5]. Available from: http://www.ibge.gov.br/home/estatistica/populacao/ condicaodevida/pof/2008_2009_analise_consumo/ pofanalise_2008_2009.pdf

26. Méjàre I, Mjör IA. Glass ionomer and resin-based fissure sealants: a clinical study. Scand J Dent Res. 1990 Aug;98(4):345-50.

27. Mjör IA, Gordan VV. A review of atraumatic restorative treatment (ART). Int Dent J. 1999 Jun;49(3):127-31.

28. Vieira ALF, Zanella NLM, Bresciani E, Barata TJ, Silva SM, Machado MA, et al. Evaluation of glass ionomer sealants placed according to the ART approach in a community with high-caries experience: 1-year follow-up. J Appl Oral Sci. 2006 Aug;14(4):270-5.

29. Mickenautsch S, Yengopal V. Validity of sealant retention as surrogate for caries prevention--a systematic review. PLoS One. 2013 Oct;23;8(10):e77103.

30. Kemoli AM, Opinya GN, van Amerongen WE, Mwalili SM. Two-year survival rates of proximal atraumatic restorative treatment restorations in relation to glass ionomer cements and Postrestoration meals consumed. Pediatr Dent. 2011 May-Jun;33(3):246-51. 